\title{
SINE-GORDON SOLITONS VS. RELATIVISTIC CALOGERO-MOSER
}

\section{PARTICLES}

\author{
S. N. M. RUIJSENAARS* \\ Centre for Mathematics and Computer Science \\ P.O.Box 94079 , 1090 GB Amsterdam, The Netherlands
}

\begin{abstract}
We discuss the connection between the $N$-soliton subspace of the sine-Gordon field theory and (a specialization of) the relativistic Calogero-Moser $N$-particle systems. At the classical level this soliton-particle relation is well understood, and we summarize its main features. At the quantum level we expect a physical equivalence of the pertinent particle systems and the sine-Gordon/massive Thirring field theory. We survey the evidence for the 2-body case in some detail.
\end{abstract}

\section{Introduction}

The sine-Gordon equation

$$
\left(\partial_{y}^{2}-\partial_{t}^{2}\right) \phi=\sin \phi
$$

defines a relativistically invariant field theory that has been studied very extensively. Indeed, there are hundreds of papers that have a bearing on it, yielding information from a great many angles. In most of these papers, one of two possible interpretations of (1) is chosen, namely as a classical or as a quantum equation. Accordingly, one either views (1) as a nonlinear evolution equation for a real-valued function $\phi(t, y),(t, y) \in \mathbb{R}^{2}$, or as an interacting relativistic quantum field theory in two space-time dimensions.

Next, we recall that both at the classical and at the quantum level the notion of 'soliton' plays a crucial role. Beginning with the classical level, we illustrate the $N$-soliton solutions to (1) with Fig. 1, which depicts a 3 -soliton collision. It exhibits one of the two hallmarks of soliton scattering, namely, the conservation of momenta in the collision. The second hallmark consists in the structure of the shift of the asymptotic soliton positions (compared to a linear superposition of 1-soliton solutions): It is factorized as a sum of shifts incurred in all pair collisions.

It is nowadays viewed as a commonplace that this picture has a quantum analog. Specifically, particle annihilation and creation has been shown to be absent in the sine-Gordon quantum field theory, and in a collision the set of momenta is conserved. Moreover, the scattering operator for an $\mathrm{N}$-particle collision factorizes as a product of all pair scattering operators.

Both in the classical and in the quantum setting, many relevant quantities are explicitly known. In particular, the 'particle spectrum' (solitons, antisolitons, and their bound states-the 'breathers') and the $N$-particle scattering are known in complete

\footnotetext{
* E-mail: siru@wxs.n
} 


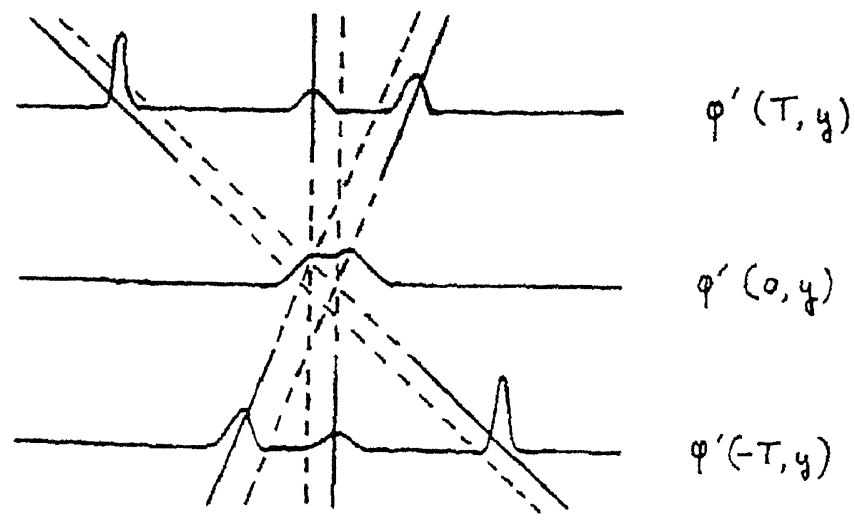

Figure 1. A 3-soliton solution to the sine-Gordon equation.

detail. The following is concerned with an appraisal of a scenario that is quite different from field theory, but which nevertheless gives rise to the same physical quantities. More specifically, this scenario originates from the question: Do there exist Hamiltonian dynamics for $N$ point particles that lead to the same factorized scattering, both at the classical and at the quantum level?

We asked this question for the first time more than 20 years ago [1]. By now, it has been answered in the affirmative and in great detail at the classical level. In this contribution we are mostly concerned with the quantum level, but as a preparation we present a short summary of features that are relevant for the classical soliton-particle correspondence.

Although there is neanwhile considerable evidence that our question has an affirmative answer at the quantum level as well, there are still some formidable road blocks for $N>2$, to which we return below. The two-body problem, however, has been completely solved, and all of our results are in accordance with the well-established lore concerning the two-body sector of the sine-Gordon quantum field theory. A considerable part of this contribution will be devoted to a survey of our two-body results.

In more detail, the plan of this paper is as follows. Section 2 concerns the classical level. Here, the gist of the particle-soliton correspondence can be casily explained in detail. For brevity we do not even sketch proofs, and we bias our account towards properties that foreshadow the state of affairs at the quantum level.

In Section 3 we first recall some highlights concerning the physical picture associated with the sine-Gordon quantum field theory. We then sketch the lines along which we expect our $N$-particle systems to lead to the same physics. As it has turned out, a key tool for our program is the functional analysis/Hilbert space theory of analytic difference operators, which is in its infancy at the present time. Pliysical heuristics based on experience with differential and discrete difference operators can casily lead to wrong answers in this area, so that a rigorous approach appears indispensable.

In Section 4 we sketch some of our results pertinent to the two-body sector of the sine-Gordon quantum field theory. As in previous sections, the rolation to the sine- 
Gordon theory involves a specialization of parameters, and is is far more natural from the 'particle viewpoint' to study the general case. We expect that other specializations of the particle systems will turn out to be relevant for a similar description of various relativistic quantum field theories of Toda type, but we restrict our attention to the sine-Gordon field theory in this contribution.

\section{The classical particle-soliton correspondence}

It transpired some 15 years ago that the point particle systems yielding a positive answer to the above question at the classical level are relativistic generalizations of the nonrelativistic Calogeru-Moser $N$-particle systems. More precisely, one needs a special class of the former, but for later purposes it is convenient to review first the most general class.

To this end we begin by recalling the most general Galilei-invariant Calogero-Moser systems. They are defined by the Hamiltonian

$$
H_{\mathrm{nr}} \equiv \frac{1}{2 M} \sum_{j=1}^{N} p_{j}^{2}+\frac{g^{2}}{M} \sum_{1 \leq j<k \leq N} p\left(x_{j}-x_{k}\right),
$$

where $p(x)$ is the Weierstrass $p$-function. Supplementing $H_{\mathrm{n}}$ with the total momentum

$$
P_{\mathrm{nr}} \equiv \sum_{j=1}^{N} p_{j}
$$

and the boost generator

$$
B \equiv-M \sum_{j=1}^{N} x_{j}
$$

one obtains a representation of the Lie algebra of the Galilei group:

$$
\left\{H_{\mathrm{nr}}, P_{\mathrm{nr}}\right\}=0, \quad\left\{H_{\mathrm{nr}}, B\right\}=P_{\mathrm{nr}}, \quad\left\{P_{\mathrm{nr}}, B\right\}=N M .
$$

The relativistic generalization of these systems is given by the time and space translation generators

$$
\begin{aligned}
& H_{\mathrm{rel}} \equiv M c^{2} \sum_{j=1}^{N} \cosh \left(\frac{p_{j}}{M c}\right) \prod_{1 \leq j<k \leq N} f\left(x_{j}-x_{k}\right), \\
& P_{\mathrm{rel}} \equiv M c \sum_{j=1}^{N} \sinh \left(\frac{p_{j}}{M c}\right) \prod_{1 \leq j<k \leq N} f\left(x_{j}-x_{k}\right),
\end{aligned}
$$

where

$$
f(x) \equiv\left(1+\frac{g^{2}}{M^{2} c^{2}} p(x)\right)^{1 / 2}
$$

and the boost generator $B(4)$. Specifically, one has the Poincare Lie algebra representation

$$
\left\{H_{\mathrm{rel}}, P_{\mathrm{rel}}\right\}=0, \quad\left\{H_{\mathrm{rel}}, B\right\}=P_{\mathrm{ar}}, \quad\left\{P_{\mathrm{nr}}, B\right\}=H_{\mathrm{rel}} / c^{2} .
$$


As should be the case, it contracts to the Galilei representation (5) in the nonrelativistic limit $c \rightarrow \infty$, since one has

$$
\lim _{c \rightarrow \infty}\left(H_{\mathrm{rel}}-N M c^{2}\right)=H_{\mathrm{nr}}, \quad \lim _{c \rightarrow \infty} P_{\mathrm{rel}}=P_{\mathrm{nr}}
$$

The only one of the above equations whose verification is nontrivial is the Poisson commutativity of $H_{\text {rel }}$ and $P_{\text {rel }}$ (space-time translation invariance). It amounts to a sequence of functional equations for the $p$-function. These equations were proved in Ref. [2], where the relativistic systems were presented for the first time.

Turning to the relation with the sine-Gordon theory, we should first of all specialize from elliptic functions to hyperbolic functions. It is convenient to work with the function

$$
f(x) \equiv\left(1+\sin ^{2} \tau / \sinh ^{2}(\mu x / 2)\right)^{1 / 2}, \quad \tau \in(0, \pi / 2],
$$

and the phase space

$$
\Omega \equiv\left\{(x, p) \in \mathbb{R}^{2 N} \mid x \in G\right\}
$$

where $G$ is the configuration space

$$
G \equiv\left\{x \in \mathbb{R}^{N} \mid x_{N}<\cdots<x_{1}\right\}
$$

Denoting a point $(x, p) \in \Omega$ by $u$, we may let $u$ evolve with the 2-parameter Hamiltonian flow $\exp \left(t H_{\text {rel }}-y P_{\text {rel }}\right)$. Then we define functions

$$
u_{j}(t, y) \equiv\left(\exp \left(t H_{\text {rel }}-y P_{\text {rel }}\right)(u)\right)_{j}, \quad j=1, \ldots, N
$$

so that $u_{j}(0,0)=x_{j}$.

We are now prepared to detail the connection to sine-Gordon solitons. First, the sine-Gordon theory corresponds to the choice $\tau=\pi / 2$ in (11). (Different $\tau$-choices yield relations to other soliton equations, cf. below.) Second, in (1) no scale parameters occur, and accordingly we need a special choice for our scale parameters $M, c$ and $\mu$ to make contact with (1), viz., $M=c=\mu=1$.

With these specializations in force, we introduce the function

$$
\phi(t, y) \equiv 4 \sum_{j=1}^{N} \operatorname{Arctg}\left(\exp \left[u_{j}(t, y)\right]\right) .
$$

It is by no means obvious, but true that $\phi(t, y)$ is an $N$-soliton solution to (1). Furthermore, one obtains all $N$-soliton solutions by letting $u$ vary over $\Omega(12)$. Finally, the requirement $u_{j}(t, y)=0$ gives rise to uniquely determined space-time trajectories $y_{j}(t)$ for the $N$-soliton collision. Some of their features can be gleaned from Fig. 2, which should be compared to Fig. 1 .

To appreciate the caption of Fig. 2, observe that the state of affairs gives rise to an intuitive picture of space as an elastic medium, which hides the goings-on in an interaction process. Although it shows the presence of individual partners for long times, it does not reveal their identities and whether they are attracted or repelled by each other.

The presence of antisolitons and bound states of solitons and antisolitons (breathers) in the sine-Gordon theory can be taken into account as well. Indeed, some of the phase space positions $x_{j}$ can be shifted to $x_{j}+i \pi / \mu$, so that repulsive interactions 


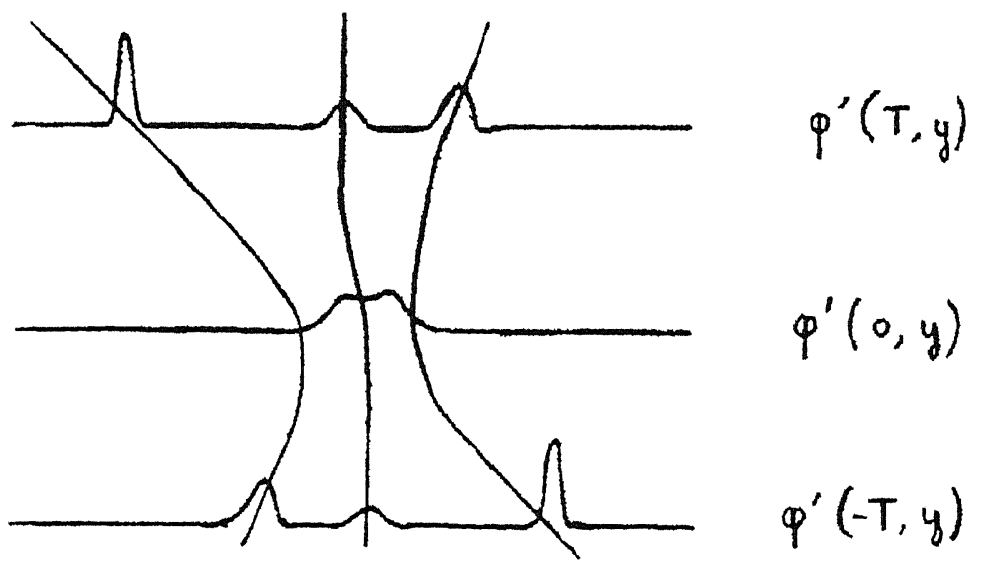

Figure 2. 'Space is a blanket.'

$\sinh ^{-2}\left(\mu\left(x_{j}-x_{k}\right) / 2\right)$ turn into attractive interactions $-\cosh ^{-2}\left(\mu\left(x_{j}-x_{k}\right) / 2\right)$. To explain the gencral situation would take us too far afield, however. (It is treated at length in Ref. [3].)

As a final remark on the physical aspects of the soliton-particle correspondence just sketched, we mention that the relation is equivariant under the Poincare group. To be quite precise, we have two actions of the latter, namely one on $\Omega$ via the flows generated by $H_{\text {rel }}, P_{\text {rel }}$ and $B$, and one on space-time $\mathbb{R}^{2}$ via the well-known freshman formulas. We have also defined a map from points $u \in \Omega$ to soliton space-time diagrams such as Fig. 2. Now the action of a Poincare transformation $g$ on a space-time picture corresponding to $u \in \Omega$ yields the space-time picture corresponding to $g(u) \in \Omega$.

We continue by describing additional features of the above relativistic CalogeroMoser systems, especially inaswuch as they yield insight into the expected quantum situation. First of all, the insistence on relativistic invariance already singles out the $\wp$-function interaction. By contrast, in (2) one can replace the pair potential $\wp(x)$ by any function $V(x)$ without losing Galilei invariance. But the potential $\wp(x)$ has the extremely restrictive property that it is not only confining, but also gives rise to a Liouville integrable system. As it turns out, this property persists for the relativistic generalization. Indeed, the Poisson commuting Hamiltonians can be more easily understood than their nonrelativistic counterparts.

To be specific, the former can be taken to be

$$
S_{l}(x, p)=\sum_{\substack{I \subset\{1, \ldots, N\} \\|I|=l}} \exp \left(\sum_{i \in I} \frac{p_{i}}{M c}\right) \prod_{\substack{j \in I \\ k \notin I}} f\left(x_{j}-x_{k}\right), \quad l=1, \ldots, N,
$$

with $f(x)$ given by (8). Note that this entails

$$
S_{N}(x, p)=\exp \left(\sum_{i=1}^{N} p_{i} / M c\right)
$$




$$
\begin{gathered}
S_{k}(x,-p)=S_{N-k}(x, p) / S_{N}(x, p), \quad k=1, \ldots, N-1, \\
H_{\mathrm{rel}}=M c^{2}\left(S_{1}(x, p)+S_{1}(x,-p)\right) / 2, \quad P_{\mathrm{rel}}=M c\left(S_{1}(x, p)-S_{1}(x,-p)\right) / 2 .
\end{gathered}
$$

The involutivity of these Hamiltonians can be reduced to a set of functional equations for the $\wp$-function [2]. These equations can be obtained as a corollary of a set of functional equations for the Weierstrass $\sigma$-function, which encode quantum commutativity [4].

The functions $S_{l}(x, p)$ are proportional to the symmetric functions of an $N \times N$ Lax matrix $L(x, p)$ that irvolves the $\sigma$-function $[5,4]$. Its hyperbolic specialization is of decisive importance in the construction of an action-angle map

$$
\Phi: \Omega=G \times \mathbb{R}^{N} \rightarrow \hat{\Omega}=\mathbb{R}^{N} \times G, \quad(x, p) \mapsto(\hat{x}, \hat{p}) .
$$

Here we think of the pure soliton case, i.e., all particles have the same 'charge', and hence interact via $f(x)$ (11). This map is constructed in Ref. [6]. It has a striking property we refer to as self-duality: The map is essentially an involution. (This duality property and related ones can also be explained in group-theoretic terms. See the recent paper Ref. [7], where also related work is mentioned.)

We single out this self-duality property, since we expect that it persists at the quantum level, cf. Section 3. Similarly, the action-angle map for the general situation (which is constructed in Ref. [3]) has certain duality properties that presumably survive quantization. Before turning to quantum issues, however, let us add some more information on the classical scattering and on relations to classical soliton equations.

First, we mention that the $t \rightarrow \pm \infty$ asymptotics of the $H_{\text {rel }}$-llow is given by

$$
\begin{gathered}
p_{N-j+1}^{j}(t) \sim \hat{p}_{j}, \\
x_{N-j+1}^{j}(t) \sim \hat{x}_{j}+c t \sinh \left(\hat{p}_{j} / M c\right) \pm\left(\sum_{k>j}-\sum_{k<j}\right) \delta\left(\hat{p}_{j}-\hat{p}_{k}\right), \\
\delta(p) \equiv \frac{1}{2 \mu} \ln \left(1+\frac{\sin ^{2}(\tau)}{\sinh ^{2}(p / 2 M c)}\right) .
\end{gathered}
$$

This is the 'particle version' of soliton scattering: The asymptotic momenta are conserved in the collision, and the position shifts are factorized as a sum of pair shifts. Exploiting the above particle-soliton correspondence, one can in fact obtain the asymptotics of sine-Gordon solutions with an arbitrary number of solitons, antisolitons and breathers in great detail [3].

Next, we make some remarks on other applications of the classical relativistic Calogero-Moser systems to soliton equations. To begin with, the $\tau=\pi / 2$ shift in (22) has more applications than just the soliton scattering in the sine-Gordon theory. Indeed, when suitable variables are employed, it also describes the scattering of solitons for the $\mathrm{KdV}$, modified $\mathrm{KdV}$ and finite-density nonlinear Schrödinger equations. Likewise, it applies to the solitons in the infinite Toda lattice, and in its relativistic $[8,9]$ and nonlocal [10] generalizations.

The $r=\pi /(n+1), n=2,3, \ldots$ shift can be used to encode the soliton scattering for the equations arising from the $A_{n}^{(1)}$-reductions of the KP and 2D Toda hicrarchies. Just as in some of the $\tau=\pi / 2$ cases, the space-time dependence of the solitons can also be tied in with suitably chosen Hamiltonian flows in the particle systems. 
At the elliptic level there is a special case of the systems (generalizing the $\tau=\pi / 2$ choice for the hyperbolic level), which is relevant for the solitons of the Landau-Lifshitz equation [11]. For the details of this case and several hyperbolic cases we refer to our survey Ref. [12]. See also Ref. [13] for more recent examples.

We conclude our sketch of the classical aspects of the particle-soliton correspondence by mentioning our lecture notes Ref. [14], where far more information on the classical particle systems is presented in a rather leisurely fashion. It might also be consulted to provide more background for the quanturn aspects, to which we now proceed.

\section{The general scenario at the quantum level}

We begin by reviewing some standard lore concerning the quantum sine-Gordon field theory, in a form that suits our later requirements. As is customary on the quantum level, we choose $c=\hbar=1$. The sine-Gordon Hamiltonian is then given by

$$
H_{\mathrm{sG}}=\int d y:\left(\frac{1}{2} \phi_{t}^{2}+\frac{1}{2} \phi_{y}^{2}+\frac{\mu^{2}}{\beta^{2}}(1-\cos \beta \phi)\right):,
$$

where the colons denote normal ordering. We recall first that it is physically equivalent to the massive Thirring model, whose Hamiltonian reads

$$
H_{\mathrm{MT}}=\int d y:\left(\Psi^{*}\left(\begin{array}{cc}
-i \partial_{y} & M \\
M & i \partial_{y}
\end{array}\right) \Psi+\frac{\lambda}{2} J^{\nu} J_{\nu}\right):
$$

The equivalence was established in well-known papers by Coleman and Mandelstam. It says that when the parameters in the two theories are related by

$$
M=\frac{8 \mu}{\beta^{2}}\left(1-\frac{\beta^{2}}{8 \pi}\right), \quad 1+\frac{\lambda}{\pi}=\frac{4 \pi}{\beta^{2}},
$$

then the models give rise to the same particle spectrum and scattering. Moreover, suitable correlation functions in the two models coincide.

The interpretation of the particles is however quite different in the two models. The massive Thirring model is a theory of interacting Dirac fermions and antifermions. It becomes free for $\lambda=0$, and it has fermion-antifermion bound states for $\lambda>0$, whereas no bound states occur for $\lambda<0$. In the sine-Gordon theory the fermions and antifermions are viewed as solitons and antisolitons, and the bound states are viewed as quantized breathers.

There appears to be less agreement on the tenet that the lowest energy bound state is to be viewed as the fundamental (neutral, bosonic) sine-Gordon particle, in relation to which the solitons and antisolitons are coherent states. In any event, the equivalence (26) entails that for $\beta^{2}>4 \pi$ the sine-Gordon Hamiltonian (1) solely describes solitons and antisolitons. It is also to be noted that $M>0$ entails $\beta^{2}<8 \pi$, and that the choice $\beta^{2}=4 \pi$ yields the free Dirac theory.

The bound state spectrum is explicitly given by the well-known Dashen-HasslacherNeveu (DHN) formula: Their rest masses read

$$
m_{n}=2 M \sin (n+1) \alpha, \quad \alpha \equiv \mu / 2 M, \quad n+1=1,2, \ldots<\pi / 2 \alpha .
$$

The DHN formula is corroborated by the explicit $S$-matrix, which was first presented by Zamolodchikov. (A review can be found in Ref. [15].) We only quote here the two-body 
amplitudes involving solitons and/or antisolitons. Denoting their asymptotic rapidity difference by $\theta$, these amplitudes can be written in terms of the function

$$
u_{S G}(\theta)=\exp \left(i \int_{0}^{\infty} \frac{d x}{x} \frac{\sinh (\alpha-\pi / 2) x}{\sinh \alpha x \cosh \pi x / 2} \sin x \theta\right)
$$

Specifically, one has

$$
\left(u_{s s}, t_{s \bar{s}}, r_{s \bar{s}}, u_{\bar{s} s}\right)(\theta)=u_{s G}(\theta)\left(1, \frac{\sinh \pi \theta / 2 \alpha}{\sinh \pi(i \pi-\theta) / 2 \alpha}, \frac{\sinh i \pi^{2} / 2 \alpha}{\sinh \pi(i \pi-\theta) / 2 \alpha}, 1\right) .
$$

(Due to fermion statistics, one gets only one amplitude for a soliton or antisoliton pair. But a soliton and an antisoliton have opposite charge, so they can be distinguished. Hence the notion of reflection and transmission coefficients makes sense in that case.)

The S-matrix involving an arbitrary number of solitons, antisolitons and bound states thereof is explicitly known as well. In case no bound states occur, it is given by sums of products of the 2-body amplitudes (29). This factorization can be performed in a consistent way, since the structure (29) entails that the Yang-Baxter equations are satisfied [15].

We do not have occasion to invoke more than the highlights just summarized. All of these date back to the period 1974-1977. Of course, in the past quarter-century a lot more information has been assembled. For example, the form factor program initiated by Karowski and co-workers has mushroomed into a minor industry. (Cf. in particular Smirnov's monograph [16], which also contains an extensive list of pertinent references.)

Let us now proceed to the quantum version of the classical relativistic CalogeroMoser systems from the previous section. Thus we should first address the question how the Poisson commuting Hamiltonians $S_{l}(16)$ should be quantized. For the case of no interaction $(f(x)=1)$ this causes no problems. Indeed, the obvious quantization of $\exp \left(p_{j} / M\right)$ is the translation

$$
\left(T_{j} \Psi\right)\left(x_{1}, \ldots, x_{j}, \ldots, x_{N}\right) \equiv \Psi\left(x_{1}, \ldots, x_{j}-i / M_{1} \ldots, x_{N}\right)
$$

into the complex plane. (Recall we have put $c=\hbar=1$.) More precisely, this operator acts on functions that have analyticity properties such that this formula has a meaning.

In particular, the $f=1$ operators are well defined on meromorphic functions $\Psi(x), x \in \mathbb{C}^{N}$, yielding so-called analytic difference operators $(A \triangle O s)$. There is however also a simple way to turn them into commuting self-adjoint operators on $L^{2}\left(\mathbb{R}^{N}, d x\right)$ : One need only pull back the self-adjoint operators of multiplication by

$$
\sum_{|J|=l} \exp \left(\sum_{j \in J} p_{j} / M\right)
$$

on $L^{2}\left(\mathbb{R}^{N}, d p\right)$ with Fourier transformation. The latter are already essentially selfadjoint on functions $\Psi(p) \in L^{2}\left(\mathbb{R}^{N}, d p\right)$ with compact support, and the Fourier transforms of these functions have an analytic continuation to entire functions of $x_{1}, \ldots, x_{N}$ whose constant imaginary part restrictions are square-integrable.

For $f \neq 1$ the state of affairs is vastly different. To begin with, one should find an ordering of the $x$ - and $p$-dependent factors in $S_{l}$ such that canonical quantization, together with an interpretation of the resulting operators as $A \Delta O$ s, gives rise to commuting $A \Delta O$ s. Whether or not these commuting $A \Delta O$ s can be promoted to commuting 
self-adjoint operators on a suitable Hilbert space is a problem of later concern. The ordering that does lead to commuting $A \Delta O$ s is quite nonobvious. For the elliptic case it involves the Weierstrass $\sigma$-function, and we will not detail it here. (Cf. Ref. [4] or our lecture notes Ref. [14].)

Specializing from now on to the hyperbolic case, we factorize $f(x)(11)$ as

$$
f(x)=f_{-}(x) f_{+}(x), \quad f_{ \pm}(x) \equiv\left(\sinh \frac{\mu}{2}\left(x \pm \frac{i g}{M}\right) / \sinh \frac{\mu}{2} x\right)^{1 / 2}
$$

Here we have set

$$
r=\alpha g, \quad \alpha \equiv \mu / 2 M,
$$

so that the sine-Gordon case $\tau=\pi / 2$ corresponds to

$$
g=\pi / 2 \alpha=\pi M / \mu \text {. }
$$

Now our commutative quantization reads

$$
\hat{S}_{l}=\sum_{|J|=i} \prod_{j \in J, k \notin J} f_{-}\left(x_{j}-x_{k}\right) \cdot \prod_{j \in J} T_{j} \cdot \prod_{j \in J, k \notin J} f_{+}\left(x_{j}-x_{k}\right) .
$$

The special case $\beta^{2}=4 \pi$ for which the sine-Gordon theory amounts to a free Dirac theory yields parameters

$$
M=\mu / \pi \Rightarrow \alpha=\pi / 2 \Rightarrow g=1,
$$

cf. (26). It is not hard to see that our quantization passes the corresponding comparison test. Specifically, the A $\Delta O s$ (34) are indeed 'free' for $g=1$ :

$$
\hat{S}_{l}=\sum_{|J|=l} \prod_{j \in J} T_{j}, \quad l=1, \ldots, N, \quad(g=1) .
$$

This can be checked by pushing the $f_{+}$-factors through the shifts, after which they combine with the $f$-factors to yield 1 , cf. (30).

With the commuting $A \Delta O s \hat{S}_{l}$ as a starting point, the key quantum mechanical problem is quite obvious: One must reinterpret these operators as commuting selfadjoint operators on a Hilbert space, and establish whether for $\tau=\pi / 2$ the associated physics (bound state spectrum, scattering) is that of the sine-Gordon/massive Thirring $N$-body sector.

To date, the most promising approach to this goal appears to be the following. One should discover/invent/construct (pick your favorite) joint eigenfunctions of the commuting $A \triangle O$ s with a great many special features that quite likely render them unique. These features ensure that the eigenfunctions can be used as kernel of a unitary joint eigenfunction transform, which makes it possible to reinterpret the $A \Delta O$ s as pullbacks of real-valued multiplication operators, yielding bona fide commuting selfadjoint operators.

We continue by listing the salient features for the case where all of the pair interactions are given by (11). Thus all particles have the same charge and hence repel each other. (Later on we will discuss the general case.) We should perhaps stress at the outset that the following may be viewed as a list of expectations/working hypotheses/requirements/conjectures (or, briefly, a wish list), the choice depending on taste, cogency, and the mood of the day. 
First, we may and will restrict attention to antisymmetric functions $E(x, p)$ with the properties that follow. Hence we have

$$
E\left(x_{\sigma}, p_{\tau}\right)=(-)^{\sigma}(-)^{\tau} E(x, p), \quad \sigma, \tau \in S_{N} .
$$

The joint eigenfunction property reads explicitly

$$
\hat{S}_{k}(\mu, M ; x) E(x, p)=\sum_{|J|=k} \exp \left(\sum_{j \in J} p_{j} / M\right) E(x, p), \quad k=1, \ldots, N
$$

We are making the parameter dependence explicit, since we expect $E(x, p)$ to have additional joint eigenfunction properties that involve transformations of the parameters. In detail, we expect

$$
\begin{aligned}
\hat{S}_{k}(2 \pi M, \mu / 2 \pi ; x) E(x, p) & =\sum_{|J|=k} \exp \left(\sum_{j \in J} 2 \pi p_{j} / \mu\right) E(x, p), \\
\hat{S}_{k}(1 / M, 1 / \mu ; p) E(x, p) & =\sum_{|J|=k} \exp \left(\sum_{j \in J} \mu x_{j}\right) E(x, p), \\
\hat{S}_{k}(2 \pi / \mu, 1 / 2 \pi M ; p) E(x, p) & =\sum_{|J|=k} \exp \left(\sum_{j \in J} 2 \pi M x_{j}\right) E(x, p) .
\end{aligned}
$$

These properties, together with real-analyticity in $\mu, M$ for $\mu, M \in(0, \infty)$, should yield a function $E(x, p)$ that is uniquely determined up to a multiplicative constant. The expected asymptotics we detail next fixes this constant. It reads

$$
\begin{aligned}
E(x, p) \sim & (2 \pi)^{-N / 2}(N !)^{-1 / 2} \sum_{\sigma \in S_{N}}(-)^{\sigma} \prod_{i<j, \sigma(i)<\sigma(j)} u\left(\frac{p_{i}-p_{j}}{2 M}\right)^{1 / 2} \\
& \times \prod_{i<j, \sigma(i)>\sigma(j)} u\left(\frac{p_{i}-p_{j}}{2 M}\right)^{-1 / 2} \exp \left(i x \cdot p_{\sigma-1}\right),
\end{aligned}
$$

for $x_{1}-x_{2}, \ldots, x_{N-1}-x_{N} \rightarrow \infty$, which entails an $S$-matrix of soliton type,

$$
S=\prod_{1 \leq i<j \leq N} u\left(\left(p_{i}-p_{j}\right) / 2 M\right)
$$

Provided

$$
\tau \in(0, \pi+\alpha)
$$

the $u$-function occurring here is given by

$$
u(z)=\exp \left(2 i \int_{0}^{\infty} \frac{d y}{y} \frac{\sinh (\alpha-\tau) y \sinh (\pi-\tau) y}{\sinh \pi y \sinh \alpha y} \sin 2 y z\right), \quad z \in \mathbb{R} .
$$

We will return to the $u$-function in Section 4.

The final item in our pure soliton wish list is that when (14) holds true, the eigenfunction transform

$$
\varepsilon: L_{a}^{2}\left(\mathbb{R}^{N}, d p\right) \rightarrow L_{a}^{2}\left(\mathbb{R}^{N}, d x\right), \quad \Psi(p) \mapsto \int_{\mathbb{R}^{N}} d p E(x, p) \Psi(p)
$$


is unitary, where the suffix denotes the antisymmetric subspace. (The unitarity constraint (44) is in particular satisfied for the sine-Gordon case $\tau=\pi / 2$.)

There are only two parameter choices for which an $N$-particle eigenfunction with all of the above properties is known, namely $\tau=\pi$ and $\tau=\alpha$. Indeed, as is readily verified, the $A \triangle O$ s are free in that case (cf. (36)). Thus we can choose

$$
E(x, p)=(2 \pi)^{-N / 2}(N !)^{-1 / 2} \sum_{\sigma \in S_{N}}(-)^{\sigma} \exp \left(i x \cdot p_{\sigma}\right)
$$

so that the operator $\varepsilon$ is a generalized sine transform.

Let us now motivate the above list. A crucial input for some of the expectations comes from the classical level. Indeed, the action-angle transform is the classical analog of the joint eigenfunction transform. Now in Ref. [6] we showed that it has factorized asymptotics deep inside the 'Weyl chamber' $G$ (13). Hence one expects factorized asymptotics for the kernel of the unitary eigenfunction transform, too. Furthermore, the classical self-duality properties translate into the symmetry property (40). The remaining eigenfunction properties are not suggested by classical results. Rather, they arise from our $N=2$ results, where all properties are valid (as we will sketch in Section 4).

Next, we comment on the joint eigenfunction properties (39) and (41). It is important to appreciate why they make sense. The point is that the coefficients of the $A \triangle O s$ in (38) and in (39) have period $2 \pi i / \mu$ and $i / M$, respectively, so that the $A \Delta O$ s commute. Likewise, the $A \Delta O$ s in (40) and (41) commute. (No such extra commutativity arises at the classical level: The function $\exp (p)$ does not Poisson commute with any nonconstant function $F(x)$.) But it should be emphasized that there are no theorems known from which one can conclude that a nontrivial function $E(x, p)$ with the properties (38)-(41) exists.

We proceed with a brief consideration of the general case, where some of the pair interactions are attractive. Physically speaking, we are then dealing with $N_{+}$particles having positive charge and $N_{-}$particles having negative charge. We expect a rather baroque picture for $N_{+} N_{-}>0$, in analogy with the classical situation and with the $N_{+} N_{-}>0$ sectors of the sine-Gordon quantum field theory.

To be more specific, let us first assume $g \in(0,1)$. This should be the simplest case to handle, since no bound states are expected. On the other hand, the scattering described via the joint eigenfunction transform should be factorized in terms of 2-body amplitudes

$$
\left(u_{++}, t_{+-}, r_{+-}, u_{--}\right)(\theta)=u(\theta / 2)\left(1, \frac{\sinh \pi \theta / 2 \alpha}{\sinh (i \pi g-\pi \theta / 2 \alpha)}, \frac{\sinh i \pi g}{\sinh (i \pi g-\pi \theta / 2 \alpha)}, 1\right) .
$$

(Here $\theta$ denotes the rapidity difference $\theta_{1}-\theta_{2}=\left(p_{1}-p_{2}\right) / M=2 p / M$.) Hence one gets a nonzero reflection, causing complications not present for $N_{+} N_{-}=0$.

For $g>1$, however, we expect a far more elaborate state of affairs. Indeed, bound states do exist for $g>1$ and $N_{+}=N_{-}=1$. Their rest masses are given by the formula

$$
m_{n}=2 M \cos (\alpha(g-n-1)), \quad n+1=1,2, \ldots<g .
$$

It should be noted that this reduces to the DHN formula (27) for the sine-Gordon case $\tau=\pi / 2, g=\pi / 2 \alpha$. Likewise, the 2 -body amplitudes (48) reduce to the sine-Gordon amplitudes (29). 
Before turning to a close-up of the 2-body case, let us comment on the viability of the general program to describe the physics of 2-dimensional relativistic soliton quantum field theories via relativistic $N$-body quantum mechanics. Indeed, there still appears to be a widespread belief that such a description cannot exist. Such a 'no-go' assertion occurs, for example, on p. 258 of the review by the Zamolodchikovs [15], and on p. 1015 of a paper by Braden and Sasaki [17].

Now for the case at hand this issue is still unresolved, on two counts. First, we have not shown yet that the above wish list materializes for $N>2$. Second, in spite of the wealth of ingenious and quite convincing sine-Gordon/massive Thirring results assembled in a great many papers, the existence of a Wightman field theory yielding the above $S$-matrix via the Haag-Ruelle theory has not been proved yet.

To refute a no-go claim, however, one counterexample suffices. At present the only counterexample we are aware of is the (fermionic) Federbush model. Specifically, this is a quantum field theory for which all Wightrnan axioms have been proved, including a verification of the solitonic $S$-matrix [18]. (To date, this model is the only Wightman field theory for which asymptotic completeness has been shown.) But the same physics can also be obtained via a certain (sequence of) $N$-body relativistic quantum mechanics [19], yielding a realization of the above program, hence a counterexample.

\section{The quantum two-body problem}

Consider the $N=2$ case of the Hamiltonians $S_{l}$ (16). Using sum and difference variables

$$
x \equiv x_{1}-x_{2}, \quad X \equiv\left(x_{1}+x_{2}\right) / 2,
$$

they read

$$
S_{1}=\exp \left(-i \partial_{X} / 2 M\right) H_{r}, \quad S_{2}=\exp \left(-i \partial_{X} / M\right),
$$

where $H_{\tau}$ is the reduced Hamiltonian

$$
H_{\tau}=f_{-}(x) \exp \left(-i \partial_{x} / M\right) f_{+}(x)+f_{+}(x) \exp \left(i \partial_{x} / M\right) f_{-}(x) .
$$

The nontrivial part of the joint Schrödinger equation

$$
\begin{aligned}
& \left(S_{1} \mathcal{F}\right)\left(x_{1}, x_{2}, p_{1}, p_{2}\right)=\left(\exp \left(p_{1} / M\right)+\exp \left(p_{2} / M\right)\right) \mathcal{F}\left(x_{1}, x_{2}, p_{1}, p_{2}\right) \\
& \left(S_{2} \mathcal{F}\right)\left(x_{1}, x_{2}, p_{1}, p_{2}\right)=\exp \left(p_{1} / M+p_{2} / M\right) \mathcal{F}\left(x_{1}, x_{2}, p_{1}, p_{2}\right),
\end{aligned}
$$

can therefore be obtained by introducing

$$
p \equiv\left(p_{1}-p_{2}\right) / 2, \quad P \equiv p_{1}+p_{2},
$$

and setting

$$
\mathcal{F}\left(x_{1}, x_{2}, p_{1}, p_{2}\right)=\exp (i P X) F(x, p) .
$$

Indeed, this entails we need only study the reduced Schrödinger equation

$$
H_{\tau} F(x, p)=2 \cosh (p / M) F(x, p) .
$$

Written out, this yields a so-called analytic difference equation (A $\Delta E$ ),

$$
\begin{aligned}
f_{-}(x) f_{+}(x-i / M) F(x-i / M, p) & +f_{+}(x) f_{-}(x+i / M) F(x+i / M, p)= \\
& =\left(e^{p / M}+e^{-p / M}\right) F(x, p) .
\end{aligned}
$$


We continue with a few remarks concerning ordinary linear second-order $A \triangle E$ s, of which $(57)$ is a concrete example. First of all, there is no detailed existence theory for such equations (as opposed to differential or discrete difference equations). Obviously, a solution in any reasonable sense should have sufficient analyticity for the shifts into the complex plane to have a clear-cut meaning. Once such a solution $F(x, p)$ exists, however, one immediately obtains an infinite-dimensional solution space. Indeed, when one multiplies $F(x, p)$ by any function $m(x, p)$ that is meromorphic in $x$ with period $i / M$, one gets another solution.

A closely related problem is the absence of a well-developed Hilbert space theory for A $\Delta$ Os. For differential and discrete difference operators the Weyl-Titchmarsh-Kodaira theory yields the link between eigenfunctions and Hilbert space features, but to date no version of this theory exists for $A \triangle O$ s. As a matter of fact, from concrete examples one sees that the Hilbert space theory of $A \Delta O$ s gives rise to some novel phenomena [20].

It is both convenient and illuminating to begin our account of explicit solutions to the Schrödinger $\mathrm{A} \triangle \mathrm{E}(57)$ by focusing on the $x \rightarrow \infty$ asymptotics. This will lead us to some important ingredients, and in particular to our 'hyperbolic gamma function'-the building block for a function $E(x, p)$ with all of the above properties. First, we explain how the explicit formula (45) arises from other items on our list. The asymptotics (42) reduces to

$$
E(x, p) \sim(2 \pi)^{-1 / 2}(-i)\left[u(p / M)^{1 / 2} e^{i x p}-u(p / M)^{-1 / 2} e^{-i x p}\right], \quad x \rightarrow \infty
$$

for the 2-body case under consideration. (The phase $-i$ is included for normalization purposes.) Now $E(x, p)$ should not only solve the $\mathrm{A} \triangle \mathrm{E}(57)$, but also the dual equation arising from (40). Using a new parameter

$$
\nu \equiv \mu / 2
$$

which minimizes factors of 2 , this equation reads

$$
\hat{f}_{-}(p) \hat{f}_{+}(p-i \nu) F(x, p-i \nu)+\hat{f}_{+}(p) \hat{f}_{-}(p+i \nu) F(x, p+i \nu)=\left(e^{\nu x}+e^{-\nu x}\right) F(x, p)
$$

with the dual interaction functions given by

$$
\hat{f}_{1}(p) \equiv(\sinh ((p \pm i \nu g) / M) / \sinh (p / M))^{1 / 2}
$$

Taking $x \rightarrow \infty$ in (60) and using (58), we can compare leading terms to deduce that the $u$-function should solve the first-order $A \triangle E$

$$
\frac{u(p / M+i \nu / 2 M)}{u(p / M-i \nu / 2 M)}=\hat{f}_{-}^{2}(p+i \nu / 2) \hat{f}_{+}^{2}(p-i \nu / 2) .
$$

Just as for differential equations, it is much simpler to solve first-order $A \Delta E_{s}$ than to solve second-order A $\triangle \mathrm{Es}$. ('Solving' stands here for 'explicitly solving'-admittedly still a somewhat imprecise expression.) In particular, the $A \Delta E(62)$ can be solved, and this yields the function (45) for the parameter range (44), as we will detail shortly.

We should emphasize at this point that the solutions to (62) form an infinitedimensional space, for the same reason as we have already explained in connection with (57). The periodic multiplier ambiguity can be removed for first-order $A \Delta E$ of a special type that is relevant here by insisting on the "optimal' analyticity and asymptotics that is compatible with the $A \triangle E$. We have made this precise in Ref. [21], and dubbed the corresponding solutions 'minimal' solutions. 
We recall that this term is also used in exact $S$-matrix theory, for instance as applied to the sine-Gordon $S$-matrix. In the latter framework, however, there are different (albeit related) requirements to meet. In particular, one insists on crossing symmetry-a notion that plays no role in our paper Ref. [21], and which is in fact a quite dispensable ingredient for the relativistic quantum mechanics we are seeking. (But of course it becomes crucial as soon as the relativistic particle systems are used to model/replace the physics of a crossing-symmetric relativistic soliton quantum field theory.)

Let us now consider the $A \triangle E(62)$. Assume we have a solution $G\left(a_{+}, a_{-} ; z\right)$ to

$$
\frac{G\left(z+i a_{-} / 2\right)}{G\left(z-i a_{-} / 2\right)}=2 \cosh \left(\pi z / a_{+}\right), \quad a_{+}, a_{-}>0
$$

available. Then we can solve (62) by setting

$$
u(z)=\frac{G(\pi, \alpha ; z-i \tau+i(\pi+\alpha) / 2) G(\pi, \alpha ; z+i \tau-i(\pi+\alpha) / 2)}{G(\pi, \alpha ; z-i(\pi-\alpha) / 2) G(\pi, \alpha ; z+i(\pi-\alpha) / 2)}
$$

as is easily verified. Now the minimal solution method introduced in Ref. [21] applies to the $A \triangle E(63)$, and it gives rise to a meromorphic function admitting the integral representation

$$
G\left(a_{+}, a_{-} ; z\right)=\exp \left(i \int_{0}^{\infty} \frac{d y}{y}\left(\frac{\sin 2 y z}{2 \sinh a_{+} y \sinh a_{-} y}-\frac{z}{a_{+} a_{-} y}\right)\right)
$$

for $|\operatorname{Im} z|<\left(a_{+}+a_{-}\right) / 2$. Therefore the integral representation (45) follows from (64) and (65).

We have called the function (65) the 'hyperbolic gamma function' for reasons explained in Ref. [21]. Observe that it is symmetric in $a_{+}, a_{-}$, so that it also solves (63) with $a_{+}$and $a_{-}$interchanged. As it has turned out, this function is not new: It is essentially equal to Kurokawa's double sine function, which in turn is a quotient of Barnes' double gamma functions. As such, it dates back more than a century. (See Refs. [22, 23] for more bibliographic information.)

A closely related function, called the 'quantum dilogarithm', was also introduced in the mid-nineties by Faddeev, cf. Ref. [24] (as we learned from R. Kashaev). It differs from our function $G\left(a_{+}, a_{-} ; z\right)$ by a multiplicative factor of the form $\exp \left(c_{0}+c_{1} z+c_{2} z^{2}\right)$, and is used in particular in the quantum Liouville theory [25].

Returning to our $u$-function (64), it readily follows from the $G-\mathrm{A} \triangle \mathrm{E}(63)$ and its $\left(a_{+} \leftrightarrow a_{-}\right)$-counterpart that it is an elementary function on the lines

$$
\tau=k \alpha+l \pi, \quad k, l \in \mathbb{Z},
$$

in the $(\alpha, \tau)$ half plane. (Recall $\alpha=\mu / 2 M$, so $\alpha>0$.) It should be noted that this set of lines is dense in the half plane. Indeed, choosing $\alpha / \pi$ irrational, we obtain a dense set of $\tau$-values when we let $k$ and $l$ vary over $\mathbb{Z}$, from which our assertion follows. Fixing a line (i.e., fixing $k_{0}, l_{0} \in \mathbb{Z}$ ), it is not hard to check that the points of intersection with the remaining lines are dense on the fixed line. (Note one has $\alpha / \pi \in \mathbb{Q}$ for these intersection points.) Therefore, the set of all intersection points is already dense in the $(\alpha, \tau)$ half plane.

In Fig. 3 we have drawn some of these lines, together with some other lines of interest. The limits indicated are worked out for the $u$-function in Ref. [21]. (See also Refs. $[12,26]$ for the NLS (nonlinear Schrödinger) limit.) 


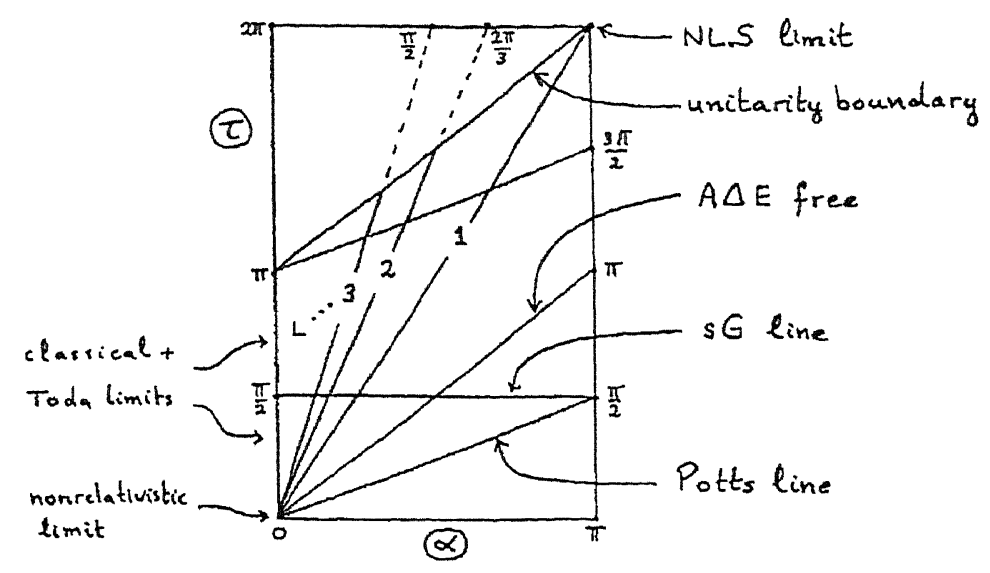

Figure 3. Special lines and limits in the $(\alpha, \tau)$ half plane.

Of special interest are the $l=0$ lines $g=L+1, L \in \mathbb{N}$, some of which are drawn in Fig. 3. On the latter $u(z)$ reduces to

$$
u(z)=(-)^{L} \prod_{k=1}^{L} \sinh (z+i k \alpha) / \sinh (z-i k \alpha) .
$$

This reflects the elementary character of our solutions to the $A \Delta \mathrm{E}(57)$, which we detail next. (They date back to Ref. [12].)

To this end we introduce the auxiliary function

$$
A(z) \equiv \prod_{ \pm k=1}^{L}[2 \sinh (z-i k \alpha)]^{-1}
$$

and the coefficient matrix

$$
\begin{aligned}
c_{k l} \equiv & (-)^{k+l} \exp (i L(L+1) \alpha / 2) \sum_{\substack{1 \leq i_{1}<\cdots<i_{k} \leq L\\
}} \exp \left(-2 i\left[i_{1}+\cdots+i_{k}\right] \alpha\right) \\
& \times \sum_{\substack{1 \\
j_{n} \notin\left\{\leq j_{1}<\cdots<j_{l} \leq L \\
-L, \ldots,-1+k, k\right\}}} \exp \left(-2 i\left[j_{1}+\cdots+j_{l}\right] \alpha\right) .
\end{aligned}
$$

Then the functions $F( \pm x, p)$ solve (57), with $F(x, p)$ given by

$$
\begin{aligned}
F(x, p) \equiv & (-i)^{L+1}[A(\nu x) A(p / M)]^{1 / 2} e^{i x p} \\
& \times \sum_{k, l=0}^{L} c_{k l} \exp [(2 k-L) \nu x+(2 l-L) p / M] .
\end{aligned}
$$

At face value, it is not at all clear that (70) solves (57) for $g=L+1 \in \mathbb{N}^{*}$. But it is important to note that once (57) is taken for granted, the dual equation (60) follows 
from $c_{k l}=c_{k k}$. This symmetry property of the matrix $c$ is not immediate either, but it is proved in Ref. [27], alongside with the eigenfunction property and asymptotics

$$
F(x, p) \sim-i u(p / M)^{ \pm 1 / 2} \exp (i x p), \quad x \rightarrow \pm \infty .
$$

The two remaining ADEs (39) and (41) on our list are readily verifed, however. Indeed, the pertinent reduced $A \triangle O$ s are given by

$$
H_{x}=\exp \left(i \pi \partial_{x} / \nu\right)+(i \rightarrow-i), \quad H_{p}=\exp \left(2 \pi M \partial_{p}\right)+(i \rightarrow-i)
$$

so the periodicity properties of the function $F(x, p) \exp (-2 x p)$ entail the desired eigenvalues $2 \cosh (\pi p / \nu), 2 \cosh (\pi M x)$.

Consider next the eigenfunction transform

$$
(\mathcal{F} \Psi)(x) \equiv(2 \pi)^{-1 / 2} \int_{\mathbb{R}} d p F(x, p) \Psi(p), \quad \Psi \in L^{2}(\mathbb{R}, d p) .
$$

It preserves parity, so it maps $L_{a}^{2}(\mathbb{R}, d p)$ into $L_{a}^{2}(\mathbb{R}, d x)$. Again, it is not obvious, but true that the restriction of $\mathcal{F}$ to $L_{a}^{2}(\mathbb{R}, d p)$ yields a unitary operator $\mathcal{E}$ (i.e., an isometry from $L_{a}^{2}(\mathbb{R}, d p)$ onto $\left.L_{a}^{2}(\mathbb{R}, d x)\right)$, provided $r \in(0, \pi+\alpha)$. Accepting this, it follows that the antisymmetric eigenfunction

$$
\begin{aligned}
E\left(x_{1}, x_{2}, p_{1}, p_{2}\right) \equiv & (2 \pi)^{-1} 2^{-1 / 2} i \exp \left[i\left(x_{1}+x_{2}\right)\left(p_{1}+p_{2}\right) / 2\right] \\
& \times\left[F\left(x_{1}-x_{2},\left(p_{1}-p_{2}\right) / 2\right)\right. \\
& \left.-F\left(x_{2}-x_{1},\left(p_{1}-p_{2}\right) / 2\right)\right]
\end{aligned}
$$

has all of the properties on the list in Section 3.

The unitarity property is proved in Ref. [20]. It is also shown there that for $\tau>\pi+\alpha$ unitarity is generically violated, in a way that is made completely explicit. Moreover, it is proved that the restriction of $F(73)$ to the even subspace $L_{s}^{2}(\mathbb{R}, d p)$ is only unitary for a certain discrete set on each line. In particular, it is not unitary for $\tau \in(0, \pi+\alpha)$.

Proceeding to the dense set of lines (66), there exist joint eigenfunctions $F( \pm x, p)$ with asympiotics (71), where $u$ is given by (64). They are elementary functions, since they equal a product of two functions of the above form (70) times a plane wave $\exp (-i x p)[27]$.

At present, it is an open question whether two joint eigenfunetions exist for arbitrary $(\alpha, \tau) \in(0, \infty) \times \mathbb{R}$, with the property that they reduce to (a multiple of) the above $F( \pm x, p)$ on the dense set of lines. But the odd combination $F(x, p)-F(-x, p)$ admits such an interpolation for $\tau \in(0, \pi+\alpha)$, and this is all that is required/expected for our list in Section 3.

In order to detail the interpolating solution, we begin by defining a 'generalized Harish-Chandra function'

$$
c\left(a_{+}, a_{-}, b_{;} z\right) \equiv G\left(a_{+}, a_{-} ; z-i b+i\left(a_{+}+a_{-}\right) / 2\right) / G\left(a_{+}, a_{-} ; z+i\left(a_{+}+a_{-}\right) / 2\right),
$$

and $a$ "weight function"

$$
w\left(a_{+}, a_{-}, b ; z\right) \equiv\left[c\left(a_{+}, a_{-}, b_{;} z\right) c\left(a_{+}, a_{-}, b_{;}-z\right)\right]^{-1} .
$$

(It is not hard to see that the function $u(z)(64)$ equals $-c(z) / c(-z)$.$) Using (65)$ and (63), one obtains

$$
w\left(a_{+}, a_{-}, b ; z\right)=4 \sinh \left(\pi z / a_{+}\right) \sinh \left(\pi z / a_{-}\right) w_{r}\left(a_{+}, a_{-}, b ; z\right)
$$


with

$$
w_{r}\left(a_{+}, a_{-}, b ; z\right)=\exp \left(\int_{0}^{\infty} \frac{d y}{y}\left(\frac{\sinh \left(a_{+}+a_{-}-2 b\right) y}{\sinh a_{+} y \sinh a_{-} y} \cos 2 y z-\frac{a_{+}+a_{-}-2 b}{a_{+} a_{-} y}\right)\right),
$$

provided

$$
b \in\left(0, a_{+}+a_{-}\right), \quad|\operatorname{Im} z|<a_{+}+a_{-}-\left|2 b-a_{+}-a_{-}\right| .
$$

Requiring (44) from now on, we set

$$
E(x, p) \equiv w(\pi, \alpha, \tau ; \nu x)^{1 / 2} R(x, p) w(\pi, \alpha, \tau ; p / M)^{1 / 2} .
$$

To define the functions on the rhs, let us first note that for real $x$ and $p$ we are entitled to invoke (77) and (78). This entails that we may view the square root functions as real-analytic odd functions on $\mathbb{R}$, which are positive for $x>0, p>0$, resp. Doing so, we turn to $R(x, p)$. This function is meromorphic and even in $x$ and $p$, and real-analytic for $x, p \in \mathbb{R}$. It is a specialization of a function $R\left(a_{+}, a_{-}, c ; v, \hat{v}\right)$ to whose definition we now proceed.

The latter is a generalization of the Gauss hypergeometric function ${ }_{2} F_{1}(a, b, c ; w)$. Just as ${ }_{2} F_{1}$ can be specialized to the Jacobi polynomials, the $R$-function admits a specialization to the Askey-Wilson polynomials. It is defined in terms of a contour integral, which generalizes the Barnes representation for the hypergeometric function. It depends on four coupling parameters $\mathrm{c} \equiv\left(c_{0}, c_{1}, c_{2}, c_{3}\right)$. Setting

$$
\begin{gathered}
s_{1} \equiv c_{0}+c_{1}-a_{-} / 2, \quad s_{2} \equiv c_{0}+c_{2}-a_{+} / 2, \quad s_{3} \equiv c_{0}+c_{3}, \quad a \equiv\left(a_{+}+a_{-}\right) / 2, \\
\hat{c}_{0} \equiv\left(c_{0}+c_{1}+c_{2}+c_{3}\right) / 2,
\end{gathered}
$$

we require at first $s_{j} \in(0, a), j=1,2,3, c_{0}, \hat{c}_{0}, v, \hat{v} \in(0, \infty)$. Then we have

$$
R\left(a_{+}, a_{-}, \mathbf{c} ; v, \hat{v}\right) \equiv \frac{1}{\left(a_{+} a_{-}\right)^{1 / 2}} \int_{\mathcal{C}} d z I\left(a_{+}, a_{-}, \mathbf{c} ; v, \hat{v}, z\right)
$$

where the integrand is given by

$$
\begin{gathered}
I\left(a_{+}, a_{-}, \mathbf{c} ; v, \hat{v}, z\right) \equiv F\left(a_{+}, a_{-}, c_{0} ; v, z\right) K\left(a_{+}, a_{-}, \mathbf{c} ; z\right) F\left(a_{+}, a_{-}, \hat{c}_{0} ; \hat{v}, z\right), \\
F\left(a_{+}, a_{-}, d ; y, z\right) \equiv\left(\frac{G\left(a_{+}, a_{-} ; z+y+i d-i a\right)}{G\left(a_{+}, a_{-} ; y+i d-i a\right)}\right)(y \rightarrow-y), \\
K\left(a_{+}, a_{-}, c_{;} z\right) \equiv \frac{1}{G\left(a_{+}, a_{-} ; z+i a\right)} \prod_{j=1}^{3} \frac{G\left(a_{+}, a_{-} ; i s_{j}\right)}{G\left(a_{+}, a_{-} ; z+i s_{j}\right)} .
\end{gathered}
$$

The contour $\mathcal{C}$ separates upward pole sequences in the $z$-plane from downward pole sequences, as depicted in Fig. 4. These doubly infinite pole sequences arise from the hyperbolic gamma functions in the integrand. (Each such sequence generalizes the well-known infinite pole sequence of Euler's gamma function $\Gamma(z)$.)

It would take us too far afield to go into the salient features of the $R$-function. It is studied in detail in Ref. [22], and in further papers to appear. We have reviewed various aspects of the $R$-function and related special functions in our lecture notes Ref. [28]. Here we only add the specialization required for the function $R(x, p)$ in $(80)$. It reads

$$
R(x, p) \equiv R(\pi, \alpha,(\tau, 0,0,0) ; \nu x, p / 2 M) .
$$




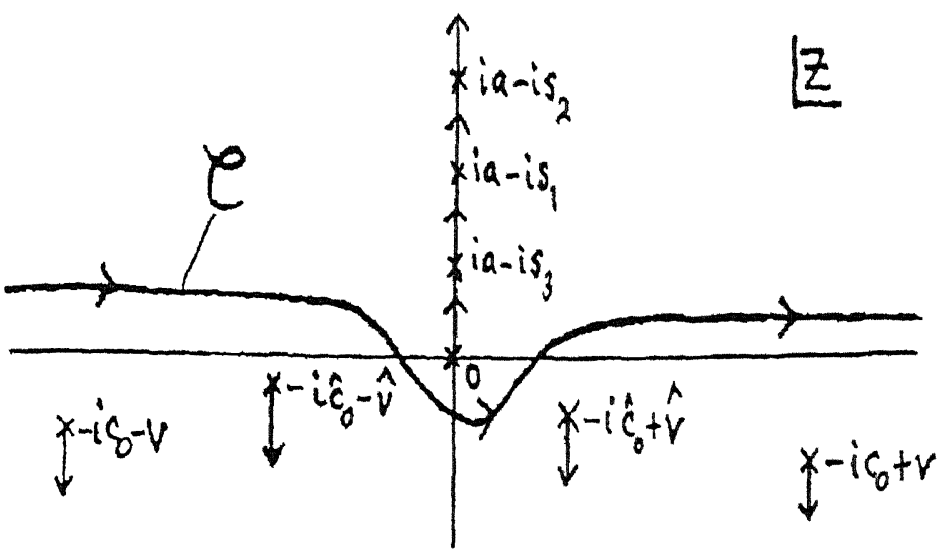

Figure 4. The pole sequences and the integration contour.

(Elsewhere we will show that the function

$$
\begin{aligned}
E\left(x_{1}, x_{2}, p_{1}, p_{2}\right) \equiv & (2 \pi)^{-1} 2^{-1 / 2} i \exp \left[i\left(x_{1}+x_{2}\right)\left(p_{1}+p_{2}\right) / 2\right] \\
& \times E\left(x_{1}-x_{2},\left(p_{1}-p_{2}\right) / 2\right)
\end{aligned}
$$

has all of the properties on the $N=2$ list, cf. Section 3 .)

Thus far, we have only dealt with the case of equal charges, where the particles repel each other. We conclude our account by sketching the state of affairs for opposite charge. (We will present the details at another occasion.) The pertinent $A \Delta E$ is then again of the form (57), but now one has

$$
f_{ \pm}(x) \equiv(\cosh (\nu x \pm i \tau) / \cosh \nu x)^{1 / 2} .
$$

First, we recall that for unitarity in the repulsive regime, we need $\tau \in(0, \pi+\alpha)$. But to obtain unitarity in the attractive regime, too, we should sharpen this to $r \in$ $(0, \pi / 2+\alpha)$. (The necessity of this restriction can already be established for the integer$g$ cases studied in Ref. [20].) Requiring this from now on, we first consider the region $g=\tau / \alpha \in(0,1]$ in the $(\alpha, \tau)$-plane, cf. Fig. 3 . Then our interpretation of the reduced 'particleantiparticle' A $\triangle \mathrm{O}$ (given by (52) and (89)) as a Hilbert space operator is such that no bound states occur. There are even and odd eigenfunctions

$$
E_{\delta}(x, p)=w_{\delta}(x)^{1 / 2} R_{\delta}(x, p) \hat{w}_{\delta}(p)^{1 / 2}, \quad \delta=e, o,
$$

whose asymptotics yields the $u$-functions

$$
u_{e}(\theta)=t_{+-}(\theta)+r_{+-}(\theta), \quad u_{o}(\theta)=t_{+-}(\theta)-r_{+-}(\theta),
$$

cf. (48). The functions $R_{e}$ and $R_{0}$ are specializations of the $R$-function (83). Specifically, one needs to choose

$$
R_{e}(x, p) \equiv R(\pi, \alpha,(0, \tau, 0,0) ; \nu x, p / 2 M)
$$




$$
R_{o}(x, p) \equiv R(\pi, \alpha,(\alpha, \tau, 0,0) ; \nu x, p / 2 M) .
$$

For brevity, we omit the definitions of the functions $w_{\delta}(x), \hat{w}_{\delta}(p), \delta=e, o$.

Turning to the regions $g \in(L, L+1), L \in \mathbb{N}^{*}$, the attractive Schrödinger $\mathrm{A} \triangle \mathrm{E}(57)$ (89) yields (in addition to the 'scattering states' (90)) $L$ pairwise orthogonal bound states with energies given by (49). The ground state reads

$$
\Psi_{0}(x)=\exp \left(\int_{0}^{\infty} \frac{d y}{y}\left(\frac{\sinh (\tau-\alpha) y \cosh \tau y}{\sinh \pi y \sinh \alpha y} \cos (2 \nu x y)-\frac{\tau-\alpha}{\pi \alpha y}\right)\right),
$$

and the excited states are then of the form

$$
\Psi_{n}(x)=P_{n}(i \sinh \nu x) \Psi_{0}(x), \quad n+1=1,2, \ldots<g,
$$

with $P_{n}(y)$ essentially a $q$-Gegenbauer polynomial. (These bound states can be obtained via (90), choosing $p$ on the imaginary axis such that $2 \cosh (p / M)$ yields the energies (49).)

With some provisos we omit, the above attractive eigenfunctions can be obtained by analytic continuation from the repulsive ones. Although this may be viewed as a form of 'crossing', we should emphasize that only for $\tau=\pi / 2$ we get the standard $S$-matrix crossing symmetry for the 2-body amplitudes (48). Indeed, as already mentioned, for $\tau=\pi / 2$ our scattering amplitudes are equal to those of the sine-Gordon theory.

As a final feature of considerable interest for the scenario of physical equivalence to the sine-Gordon/massive Thirring quantum field theories, we show that the ground state (94) can be rewritten as a quite simple function for $\tau=\pi / 2$. First, we use (65) and (63) to write

$$
\begin{aligned}
\Psi_{0}^{2}(x) & =\frac{G(\pi, \alpha ; \nu x+i \alpha / 2) G(\pi, \alpha ; \nu x-i \tau+i \alpha / 2)}{G(\pi, \alpha ; \nu x-i \alpha / 2) G(\pi, \alpha ; \nu x+i \tau-i \alpha / 2)} \\
& =4 \cosh (\nu x) \cosh (\nu x-i \tau) \frac{G(\pi, \alpha ; \nu x-i \tau-i \alpha / 2)}{G(\pi, \alpha ; \nu x+i \tau-i \alpha / 2)} .
\end{aligned}
$$

Now we note that for $\tau=\pi / 2$ we may use the $\left(a_{+} \leftrightarrow a_{-}\right)$-version of (63) to deduce

$$
\Psi_{0}(x)=[\sinh (2 \nu x) / \sinh (\pi M x)]^{1 / 2}, \quad \tau=\pi / 2 .
$$

To summarize, the elementary function (97) can be viewed as the (internal wave function of the) lowest energy bound state of the sine-Gordon soliton and antisoliton. Note that it manifestly has decay $\exp (-\nu(1-g)|x|)$ for $x \rightarrow \pm \infty$. Thus, the squareintegrability of the bound states (95) is immediate.

\section{References}

1. Ruijsenaars, S.N.M. (1980) A positive onergy dynamics and scattering theory for directly interacting relativistic particles, Ann. Phys. (N.Y.) 126, 399-449.

2. Ruijsenaars, S.N.M. and Schneider, H. (1986) A new class of integrable systems and its relation to solitons, Anr. Phys. (N.Y.) 170, 370-405.

3. Ruijsenaars, S.N.M. (1994) Action-angle maps and scattering theory for some finite-dimensional integrable systems. II. Solitons, antisolitons, and their bound states, Publ. RIMS Kyoto Univ. 30, $865-1008$

4. Ruijsenaars, S.N.M. (1987) Complete integrability of relativistic Calogero-Moser systems and elliptic function identities, Comm. Math. Phys. 110, 191-213. 
3 Bruschi, M. and Calogero, F. (1987) The Lax representation for an integrable class of relativistic dymanical systems, Comm Math. Phy. 100, 48:-492

6. Rujsenaars, S.N. M. (1988) Action-angle maps and scattering theory for some finite-dmensional integrable systems. 1. The pure soliton case, Comm. Math. Phys. 115, 127m-165.

7. Fock, V., Gorsky, A., Nekrasov, N. and Rubtsov, V. (2000) Duality in integrable syatems and gage theories, I. High En. Phys. 7, no. 28, 1-39

8. Cosentino, S. (1991) Infinita relativistic Toda lattice: seattering problem and canonical structure, Inverse Problems $7,535-555$

9. Ohta, Y., Kajiwara, K., Matsukidaira, J. and Satsuma, J. (1993) Casorati determisant solution for the relativiatic Toda lattice equation, J. Moth. Phys. 34, 5190-5204.

10. Ruijenaars, S.N.M. (2001) Reflectionless analytic difference operators II. Relations to soliton systems, J. Nonidr. Math. Phys. 8, no. 2

11. Date, E., Jimbo, M. Kashwara, M. and Miwa, T. (1983) Landau-Lifshitz equation: solitons, quasi-periodic solutions and infinite-dimensional Lie algobras, J. Phys. A16, 221-236.

12. Ruigsenaars, S.N.M. (1990) Finile-diwensional soliton systems, in B. Kupershmid" (ed.) Integrable and superintegrable systems pp. 165-206, World Scientific, Singapore.

13. Ruijsenaurs, S.N.M. (1997) Integrable particle systerns vs solutions to the KP and 2D Toda aquations, Ann. Phys. (N.Y.) 256, 220-301.

14. Ruijsenaars, S.N.M. (1999) Systems of Calogero-Moser type, in G. Semenoff and L. Vinet (eds) Procedings of the 1904 Banff summer school Particles and fields, CRM Ser. in Math. Phys., pp. 231-352, Springer, New York.

15. Zamolodchikov, A.B. and Zamolodchikov, Al.B. (1979) Factorized S-matrices in two dimensions as the exact solutions of certain relativistic quantum field theory models, Ans. Phos. (N.Y.) 120, $253-291$

16. Sminov, F.A. (1992) Form foctors in completely iniegrable models of quantum field theory, Adv. Ser. in Math. Phys., Vol. 14, World Scientific, Singapore.

17. Braden, H.W. and Sasaki, R. (1997) The Ruijsenaars-Schneider model, Progr. Theor. Phys. 97, 1003-1017.

18. Ruijsenaars, S.N.M. (1982) The Wightman axioms for the fermionic Federbush model, Comm. Math. Phys. 87, 181-228.

19. Ruijenuars, S.N.M (1980) On one-dimensional integrable quantum systems with infinitely many degrees of freedom, Ann. Phys. (N.Y.) 128, 335-361.

20. Ruijsenaars, S.N.M. (2000) Hilbert space theory for reflectionless relativistic potentials, Publ. RIMS Kyoto Univ. 36, 707-753.

21. Ruijsenaars, S.N.M. (1997) First order analytic difference equations and integrable quanturn systerns, J. Math. Phys. 38, 1069-1146.

22. Rujisenaars, S.N.M. (1999) A generalized hypergeometric function satisfying four analytic difference equations of Askey.Wilson type, Commush Math. Phys. 206, 639-690.

23. Rujsenaars, S.N.M. (2000) On Barnes' multiple zeta and garama functions, Adv. in Math. 158, $107-132$

24. Faddeev, L.D. (1995) Discrete Hoisenberg-Weyl group and modular group, Leti. Math. Phys. 34, 249-254.

25. Faddeev, L.D., Kashaev, R.M. and Volkow, A.Yu. (2000) Strongly coupled quantum discrete Liouville theory. I: Algebraie approach and duality, preprint

26. Ruijsenaars, S.N.M. (1999) Relativistic Lamé functions: the special case $g=2, J$. Phys. A: Math. Gen. 32, 1737-1772.

27. Rujjsenaars, S.N.M. (1999) Generalized Lamé functions. II. Hyperbolic and trigonometric specializations, J. Math. Phys. 40, 1627-1663.

28. Ruijsenaars, S.N.M. (2000) Special functions associated with Calogero-Moser type quantum systerns, in J. Harnad, G. Sabidussi, F. Winternitz (eds.) Proceedirgs of the 1999 Montréal Seminaire de Mathematiques Suptreares, CRM Proceedings and lecture notes, Vol. 26, pp. 189-226, Amer. Math. Soc., Providence. 